Bundesgesundheitsbl 2011 · 54:1135-1144

DOI 10.1007/s00103-011-1352-8

(c) Springer-Verlag 2011

\section{Anforderungen an die Hygiene bei Punktionen und Injektionen}

\section{Empfehlung der Kommission für Krankenhaushygiene und Infektions- prävention beim Robert Koch-Institut (RKI)}

\section{Kategorien in der Richtlinie für Krankenhaushygiene und Infektions- prävention (2010)}

Die im nachfolgenden Dokument gegebenen Empfehlungen basieren auf den aktuellen Kategorien der Richtlinie für Krankenhaushygiene und Infektionsprävention aus dem Jahr 2010. Diese werden nachfolgend nochmals aufgeführt [1].

Kategorie IA:
Diese Empfehlung basiert auf gut konzipier-
ten systematischen Reviews oder einzelnen
hochwertigen randomisierten kontrollierten
Studien.
Kategorie IB:
Diese Empfehlung basiert auf klinischen oder
hochwertigen epidemiologischen Studien
und strengen, plausiblen und nachvollzieh-
baren theoretischen Ableitungen.
Kategorie II:
Diese Empfehlung basiert auf hinweisenden
Studien/Untersuchungen und strengen,
plausiblen und nachvollziehbaren theoreti-
schen Ableitungen.
Kategorie III:
Maßnahmen, über deren Wirksamkeit nur
unzureichende oder widersprüchliche Hin-
weise vorliegen, deshalb ist eine Empfehlung
nicht möglich.
Kategorie IV:
Anforderungen, Maßnahmen und Verfah-
rensweisen, die durch allgemein geltende
Rechtsvorschriften zu beachten sind.

\section{Hintergrund}

Punktionen und Injektionen gehören zu den häufigsten invasiven Eingriffen in Krankenhäusern, Arztpraxen und in der sonstigen ambulanten Versorgung. Im medizinischen Bereich obliegt die Durchführung dabei je nach Indikation unterschiedlichen Berufsgruppen. Das Spektrum der hier behandelten Punktionen und Injektionen reicht von kurz dauernden, gering invasiven Eingriffen wie z. B. Blutentnahmen oder intrakutanen Applikationen bis hin zu tiefen Punktionen / Injektionen mit großlumigen Punktionsnadeln. Es schließt fernerhin Punktionen ein, die der Anlage einer für kurze Zeiträume liegen bleibenden Nadel (z. B. Butterfly) und der Insertion von perkutanen Kathetern dienen, sofern es sich nicht um Gefäßkatheter handelt.

Die vorstehenden Empfehlungen gelten für die Durchführung von Punktionen und Injektionen durch medizinisches Fachpersonal. Bei Durchführung solcher Eingriffe im häuslichen Bereich durch $\mathrm{Pa}$ tienten selbst, beispielsweise im Rahmen chronischer Erkrankungen, welche eine regelmäßige Selbstapplikation von Medikamenten erfordern, wird auf die Empfehlungen der medizinischen Fachgesellschaften (z. B. $[2,3]$ und www.rki.de $>$ Infektionsschutz $>$ Krankenhaushygiene $>$ FAQ > Insulingabe) verwiesen.

Neben den nachfolgenden Empfehlungen und Aussagen sind die Regeln und
Standards des Arbeitsschutzes, z. B. die TRBA 250, zu beachten.

\section{Bezug zu anderen Empfehlungen}

In den hier vorgelegten Empfehlungen ergeben sich zahlreiche Berührungspunkte zu früheren Mitteilungen in der Richtlinie für Krankenhaushygiene und Infektionsprävention. Im Einzelnen sind dies die Empfehlungen:

- Händehygiene [4],

- Prävention Gefäßkatheter-assoziierter Infektionen [5],

- Anforderungen an die Hygiene bei der Reinigung und Desinfektion von Flächen [6],

- Prävention postoperativer Infektionen im Operationsgebiet [7],

- Anforderungen der Hygiene bei Operationen und anderen invasiven Eingriffen [8],

- Anforderungen der Krankenhaushygiene und des Arbeitsschutzes an die Hygienebekleidung und persönliche Schutzausrüstung [9],

- Personelle und organisatorische Voraussetzungen zur Prävention nosokomialer Infektionen [10],

- Anforderungen an die Hygiene bei der medizinischen Versorgung von immunsupprimierten Patienten [11].

Bei der Erarbeitung von Hygieneplänen, Standardarbeitsanweisungen oder ande- 
ren in einer Einrichtung geltenden Regelungen sollten die vorgenannten Dokumente zusätzlich berücksichtigt werden.

Der nachfolgende Text enthält Empfehlungen, die sich auf die Zubereitung von Medikamenten und die Vorbereitung von Punktionen und Injektionen in patientennahen Bereichen beziehen. Ausdrücklich nicht dargestellt sind Hygienemaßnahmen, die bei der Herstellung von Parenteralia in einer zentralen Apotheke zu beachten sind. Hierzu wird auf das Arzneimittelrecht sowie auf pharmazeutische Leitlinien verwiesen $[12,13,14]$.

Besonders wichtig für alle im Folgenden dargestellten Hygienemaßnahmen ist eine sorgfältige Händehygiene entsprechend der o. g. Empfehlung [4], welche für alle manuellen Tätigkeiten im Zusammenhang mit der Vorbereitung und Durchführung von Punktionen und Injektionen einzuhalten ist.

\section{Bezug zum Arzneimittel- und Medizinprodukterecht}

Bei der Zubereitung von parenteralen Arzneimitteln zur Anwendung am $\mathrm{Pa}$ tienten sind die Herstellerinformationen zu beachten, die sich aus der Packungsbeilage und der Fachinformation ergeben. Gemäß $\$ \$ 11$ und 11a Arzneimittelgesetz (AMG) sind beide Dokumente Bestandteil der arzneimittelrechtlichen Zulassung eines Arzneimittels. Packungsbeilage und Fachinformation folgen jeweils stets der gleichen Gliederung und enthalten unter anderem Aussagen zur Art und Dauer der Anwendung, zur Lagerung, zur chemischphysikalischen Haltbarkeit nach Auflösung oder zur Mischbarkeit mit anderen Substanzen. Aus beiden Dokumenten ist auch zu entnehmen, ob das Arzneimittel vom Hersteller als Mehrdosenbehältnis deklariert ist. In diesem Fall enthalten die beiden Dokumente auch Aussagen zur maximal möglichen Lagerdauer des Arzneimittels im angebrochenen Zustand und $\mathrm{zu}$ weiteren Bedingungen der Lagerung und Mehrfachentnahme. Die sichere Anwendung eines parenteralen Arzneimittels erfordert die Einhaltung aller in den beiden Dokumenten aufgeführten Maßnahmen und Hinweise.

Sind Anwender der Auffassung, dass in der Packungsbeilage oder der Fachinfor- mation eines Arzneimittels wichtige Angaben für die Einhaltung der Infektionsprävention fehlen oder gegebene Hinweise nicht eindeutig sind, sollte dies der zuständigen Apotheke oder Krankenhausapotheke gemeldet werden. Diese informiert den Hersteller sowie die zuständige Überwachungsbehörde und die Arzneimittelkommission der Deutschen Apotheker.

Werden für Punktionen und Injektionen Medizinprodukte verwendet (z.B. Injektionsautomaten, Punktions-Sets, Portsysteme), so sind die in der Gebrauchsanweisung enthaltenen Herstellerempfehlungen für die Anwendung zu beachten.

Bei unvollständigen und/oder nicht plausiblen Angaben in der Gebrauchsanweisung von Medizinprodukten ist der Hersteller zur Präzisierung und/oder Korrektur der Angaben aufzufordern. Sofern der Hersteller (oder Vertreiber) in Deutschland ansässig ist, sollte die Überwachungsbehörde des Bundeslandes, in dem der Hersteller/Vertreiber seinen Sitz hat, nachrichtlich informiert und bei Bedarf um Unterstützung gebeten werden. Sind Hersteller oder Vertreiber nicht in Deutschland ansässig, ist das BfArM als die für solche Fälle zuständige Behörde zu informieren, damit die zuständige europäische Behörde über den Sachverhalt in Kenntnis gesetzt und um Abklärung mit dem Hersteller/Bevollmächtigten gebeten werden kann.

\section{Infektionsrisiko bei Punktionen und Injektionen}

Aus den in Tabelle 1 zusammengestellten Literaturangaben lässt sich ablesen, dass das Risiko punktionsassoziierter Infektionen von der Art und Ort des Eingriffs abhängig ist. Für das höhere Infektionsrisiko bei Punktionen von Körperhöhlen oder Organen sind vermutlich mehrere Faktoren ausschlaggebend. Zum einen können aufgrund des größeren Kanülendurchmessers Hautstanzen in den Stichkanal verschleppt werden $[15,16]$. Zum anderen ermöglicht das Fehlen natürlicher Infektabwehrmechanismen in abgegrenzten sterilen Körperhöhlen wie z. B. den Gelenkhöhlen, dem Liquorraum oder der vorderen und hinteren Augenkammer, das Entstehen einer Infektion bereits bei geringen
Erregermengen. Auch die allgemeine Abwehrlage des Patienten (z. B. Grundkrankheiten, insbesondere solche mit herabgesetzter Immunfunktion oder Gerinnungsstörungen), beeinflusst das Infektionsrisiko.

Neben den genannten Faktoren wird das Infektionsrisiko beeinflusst durch:

- die Rahmenbedingungen des Zubereitens und Aufziehens von Medikamenten (z. B. Zubereitung in einer zentralen Apotheke unter Reinraumbedingungen oder einer Zubereitung auf Station) [17], - die Rahmenbedingungen der Punktion (z. B. Notfallbedingungen, komplexe technische Abläufe bei Punktionen und Injektionen),

- bei Injektionen durch die Eigenschaften des applizierten Arzneimittels, Applikation von Substanzen, welche die Infektabwehr unterdrücken (z. B. Corticosteroide) $[18,19,20]$,

- die Erfahrung des Ausführenden, z. B. beim Legen einer perkutanen endoskopischen Gastrostomie (PEG) [21],

- die Liegedauer eines perkutan inserierten Katheters, z. B. eines Periduralkatheters oder Ventrikelkatheters [22].

Hygienefehler, d. h. die Nichtbeachtung der Prinzipien der Asepsis bei der Zubereitung von Medikamenten und ihrer Applikation bei Injektionen, haben in der Vergangenheit immer wieder zu Ausbrüchen von Infektionen durch bakterielle Erreger oder blutübertragene Viren geführt (z. B. Enterobacter cloacae, Hepatitis-B-, Hepatitis-C-Virus) [23, 24, 25, 26, 27, $28,29,30,31,32]$.

\section{Räumliche Anforderungen}

Aus hygienischer Sicht handelt es sich bei Punktionen und Injektionen bei immunkompetenten Patienten um invasive Eingriffe, die in zur Pflege oder Behandlung von Patienten geeigneten Räumen durchgeführt werden können. Eine Raumlufttechnische (RLT-) Anlage ist aus infektiologischen Gründen nicht notwendig. Die Entscheidung, in welchen Räumlichkeiten bestimmte Punktionen oder Injektionen durchgeführt werden, wird in der Regel aufgrund von Erfordernissen der medizinischen Behandlung des Patienten bzw. 


\begin{tabular}{|c|c|c|c|}
\hline Punktionsart & $\begin{array}{l}\text { Mögliche infektiöse } \\
\text { Komplikation }\end{array}$ & $\begin{array}{l}\text { Postpunktionelle Infektionsrate } \\
\text { nach Literaturangaben }\end{array}$ & $\begin{array}{l}\text { Ausgewählte } \\
\text { Referenzen }\end{array}$ \\
\hline s.c.-Injektion & Abszess, Cellulitis Phlegmone & $\begin{array}{l}\text { Einzelfälle und Fallserien beschrieben, } \\
\text { Häufigkeit unbekannt }\end{array}$ & {$[76,77,78]$} \\
\hline $\begin{array}{l}\text { s.c.-Injektion mit Katheter- } \\
\text { anlage für s.c.-Infusion }\end{array}$ & Phlegmone & $\begin{array}{l}\text { 0,1-0,4 Infektionen } \\
\text { pro Patientenjahr }\end{array}$ & {$[79,80]$} \\
\hline i.m.-Injektion & $\begin{array}{l}\text { Spritzenabszess } \\
\text { Nekrotisierende Fasziitis, } \\
\text { Septikämie }\end{array}$ & $\begin{array}{l}1: 10.000-1: 12.000 \\
1: 1.000 .000\end{array}$ & {$[20,81]$} \\
\hline Diagnostische Lumbalpunktion & purulente Meningitis & $\begin{array}{l}0: c a .28 .000 \\
\text { Einzelfälle, keine Nennerangabe }\end{array}$ & {$[82,83,84,85]$} \\
\hline Lumbalpunktion mit Einzelinjektion & purulente Meningitis & $\begin{array}{l}\text { 2:3.000 (nach Injektion von } \\
\text { Zytostatika bei Meningeosis) }\end{array}$ & [83] \\
\hline Peridurale / intraspinale Anästhesie & $\begin{array}{l}\text { purulente Meningitis, } \\
\text { epiduraler Abszess }\end{array}$ & $\begin{array}{l}1: 500.000 \\
1: 5.000\end{array}$ & {$[47,50]$} \\
\hline Gelenkpunktion & Purulente Arthritis & $\begin{array}{l}1: 25.000 \\
1: 35.000 \\
1: 3.000-1: 50.000 \\
1: 1.000-1: 16.000\end{array}$ & {$[18,86,87,88]$} \\
\hline Intravitreale Injektion & Panophthalmie & $\begin{array}{l}2: 3.000 \\
2: 1.800\end{array}$ & {$[89,90]$} \\
\hline Nierenbiopsie & Nierenabszess & $1: 1.000$ & [91] \\
\hline $\begin{array}{l}\text { Amniocentese } \\
\text { Chorionzottenbiopsie }\end{array}$ & $\begin{array}{l}\text { Aborte, auch verursacht durch } \\
\text { intrauterine Infektion }\end{array}$ & $<1: 1.000$ & {$[92,93]$} \\
\hline $\begin{array}{l}\text { Perkutane endoskopische } \\
\text { Gastrostomie (PEG) }\end{array}$ & lokale Infektion* & $\begin{array}{l}26 \% \\
24,5 \% \\
47 \% \\
33,6 \%\end{array}$ & {$[21,52,54,55]$} \\
\hline Externe Ventrikeldrainage & Meningitis & $3-11 \%$ & [22] \\
\hline
\end{tabular}

der technischen Rahmenbedingungen der Punktion getroffen. Aufgrund benötigter Geräte (z. B. Durchführung von Punktionen unter Röntgen- oder Ultraschallkontrolle) oder der Notwendigkeit, benötigtes Zubehör in unmittelbarer Nähe zum Eingriffsort zu bevorraten, werden bestimmte invasive Punktionen und Injektionen, wie beispielsweise eine Amniozentese oder die Anlage eines Periduralkatheters, üblicherweise in Behandlungs- oder Eingriffsräumen bzw. in Einleitungsräumen von OPEinheiten durchgeführt [8]. Wissenschaftliche Studien, welche die Infektionsrate bei Punktionen unter verschiedenen räumlichen Bedingungen vergleichen, existieren jedoch nicht.

Für Punktionen und Injektionen, die mehrere Vorbereitungsschritte erfordern, wird eine ausreichend große, freie Arbeitsfläche zur Bereitstellung und Herrichtung des Zubehörs benötigt. Kontaminationen solcher Arbeitsflächen durch par- tikelgebundene [33] oder wassergetragene Erreger [34] haben in der Vergangenheit zu Ausbrüchen von Injektions- bzw. Infusions-assoziierten Infektionen geführt. Daraus ergibt sich die Forderung, dass derartige Arbeitsflächen durch geeignete Vorkehrungen vor Umgebungskontamination (z. B. Sicherheitsabstand zu einem Waschbecken oder Trennwand) geschützt werden müssen [35].

\section{Empfehlungen:}

- Wenn eine Arbeitsfläche zur Vorbereitung benötigt wird, so ist diese vor Umgebungskontamination, z. B. Spritzwasser, zu schützen (Kat. II).

- Die Arbeitsfläche muss leicht zu reinigen und zu desinfizieren sein (Kat. II).

\section{Vorbereitung von Punktionen und Injektionen}

Grundsätzlich sind für alle vorbereitenden Maßnahmen die allgemein anerkannten Regeln der Standardhygiene einzuhalten (www.rki.de $>$ Infektionsschutz $>$ Krankenhaushygiene $>$ Informationen zu ausgewählten Erregern > Einleitung; [36]). Das mit der Vorbereitung von Punktionen und Injektionen betraute Personal ist regelmäßig, z. B. durch die Hygienefachkraft oder durch die sonst in einer Einrichtung Verantwortlichen, zu schulen (Es ist üblich, dass über solche Unterweisungen ein Protokoll geführt wird und die Verfahrensbeteiligten dies eigenhändig unterzeichnen.). Auch sollte die Einhaltung der nachfolgend beschriebenen Grundsätze durch Beobachtung und Bewertung der Abläufe vor Ort (z. B. durch das Hygienefachpersonal) überprüft werden [25]. 
Injektions- bzw. Infusionsflaschen und Ampullen müssen durch Sichtprüfung auf Verfärbungen, Trübungen und Defekte überprüft werden. Bei Auffällig keiten dürfen die Gefäße nicht verwendet werden. Das Ereignis ist dem Hersteller zu melden (s. o.).

Wenn die Vorbereitung des Zubehörs auf einer Arbeitsfläche erfolgt, muss diese vorher wischdesinfiziert bzw. bei Punktionen, die einen Wechsel und ein zwischenzeitliches Ablegen steriler Instrumente erfordern, zusätzlich steril abgedeckt werden. Für jeden Patienten sind eine neue sterile Spritze und Kanüle zu verwenden. (Auch Insulin-Pens müssen patientenbezogen eingesetzt werden.) Spritzen und Kanülen sind mit der Peeloff-Technik aus den Sterilverpackungen zu entnehmen bzw. bereitzustellen. Der Kolben von Spritzen darf beim mehr maligen Vor- und Zurückbewegen nur an der Stempelplattform angefasst werden, da es ansonsten zu einer Kontamination der Innenseite des Zylinders kommen kann.

Das Gummiseptum von Injektionsflaschen ist vor dem Einführen der Entnahmekanüle mit einem alkoholischen Desinfektionsmittel zu desinfizieren [25], sofern der Hersteller nicht ausdrücklich die Sterilität des Gummiseptums unterhalb des Verschlusses garantiert. Für diesen Zweck sind z. B. alkoholische Hautdesinfektionsmittel geeignet. Die Desinfektion des Gummiseptums kann durch Abwischen mit einem desinfektionsmittelgetränkten keimarmen Tupfer oder mittels Einsprühen erfolgen. Keimarm in der hier verwendeten Diktion sind Tupfer dann, wenn sie im Herstellungsprozess sterilisiert und nach Entnahme aus der Sterilgutverpackung kontaminationsgeschützt gelagert wurden. Ebenso gelten als keimarm solche, die unter qualitätskontrollierten, keimarmen Bedingungen hergestellt, mit einem geeigneten Desinfektionsmittel vorgetränkt und in einer Einzelverpackung (z. B. Staniolfolie) verpackt sind.

Das Desinfektionsmittel muss vor der Einführung der Kanüle in das Septum abgetrocknet sein. Es ist zulässig, bei kleineren Injektionsflaschen Raumluft vorzuspritzen, um die Entnahme zu erleichtern. Bei größeren Ampullen ( $\geq 50 \mathrm{ml}$ ) empfiehlt sich die Verwendung einer Spike-Mehrfachentnahmekanüle mit Luftfilter oder einer Überleitkanüle.

Bei wiederholter Entnahme aus Mehrdosenbehältnissen ist für jede Entnahme eine frische Kanüle und Spritze zu verwenden. Es ist eine Kanülengröße zu wählen, die keine Stanzöffnung hinterlässt. Die Kanüle darf bei der weiteren Lagerung nicht im Gummiseptum verbleiben, sondern ist sofort nach Gebrauch zu entfernen. Die Verwendung von Einmalkanülen ist aus Gründen der Patientensicherheit und Asepsis das optimale Vorgehen.

Das Risiko der Verwendung von Mehrfachentnahmekanülen (Spikes), die nach der Punktion im Gummiseptum von Mehrdosenbehältnissen verbleiben, kann derzeit aus Literaturdaten nicht beurteilt werden. Bei der Verwendung von Mehrfachentnahmekanülen ist $\mathrm{zu}$ beachten, dass für jede erneute Entnahme eine frische Spritze verwendet wird. In der Literatur sind schwere Infektionszwischenfälle berichtet worden, wenn gegen diesen Grundsatz verstoßen wurde $[23,37]$.

Die Zubereitung und das Aufziehen von Medikamenten soll unmittelbar vor der geplanten Applikation erfolgen, da In-vitro-Studien eine Keimvermehrung nach einer bis mehreren Stunden in Abhängigkeit von der Art des Medikamentes, vom initialen Keimeintrag und ggf. antimikrobiellen Eigenschaften von enthaltenen Zusätzen gezeigt haben $[38,39]$. Erforderliche Ausnahmen (z. B. für die Notfallmedikamente in Ambulanzen oder Arztpraxen) müssen vom Krankenhaushygieniker (ggf. dem niedergelassenen Arzt) und der zuständigen Apotheke (ggf. unter Hinzuziehung weiteren Sachverstandes in der jeweiligen Einrichtung) in einer Standardarbeitsanweisung beschrieben werden.

\section{Empfehlungen:}

- Vor dem Beginn des Herrichtens von Medikamenten und Materialien für Punktionen oder Injektionen ist eine hygienische Händedesinfektion durchzuführen (Kat. IA). Ergänzend sind die Grundsätze der Standardhygiene zu beachten (Kat. II).

- Wenn die Vorbereitung des Zubehörs auf einer Arbeitsfläche erfolgt, muss diese vorher wischdesinfiziert bzw. bei
Punktionen, die einen Wechsel und ein zwischenzeitliches Ablegen steriler Instrumente erfordern, zusätzlich steril abgedeckt werden (Kat. II).

- Die Zubereitung von Medikamenten zur Injektion soll unmittelbar vor der geplanten Applikation erfolgen (Kat. II). Erforderliche Ausnahmen müssen mit dem Krankenhaushygieniker (ggf. dem niedergelassenen Arzt) und dem zuständigen Apotheker besprochen und in einer Standardarbeitsanweisung festgelegt werden.

- Das Gummiseptum von Injektions- und Infusionsflaschen ist vor dem Einführen einer Kanüle mit einem alkoholischen Desinfektionsmittel zu desinfizieren. Eine Ausnahme stellen Gummisepten dar, bei denen der Hersteller die Sterilität unter der Abdeckung garantiert (Kat. IV).

- Werden Teilmengen aus einem Mehrdosenbehältnis mittels einer Einmalkanüle entnommen, ist für jede Entnahme eine neue Spritze und Kanüle zu verwenden. Einmalkanülen dürfen nicht im Mehrdosenbehältnis verbleiben (Kat. II).

- Werden Teilmengen aus einem Mehrdosenbehältnis mithilfe einer Mehrfachentnahmekanüle (Spike) entnommen, ist für jede Entnahme eine neue Spritze zu verwenden (Kat. II).

- Auf angebrochenen Mehrdosenbehältnissen sind das Anbruchdatum und die Verwendungsdauer zu vermerken (Kat. IV).

- Das Personal ist regelmäßig in den hygienischen Arbeitstechniken zu schulen (Die Unterweisungen sind zu dokumentieren.) Der Erfolg der Schulungen sollte durch Beobachtung und Bewertung der Abläufe vor Ort, z. B. durch das Hygienefachpersonal, überprüft werden (Kat. II).

\section{Durchführung von Punktionen und Injektionen}

Auswahl der Punktionsstelle: Das Punktionsareal bzw. die Injektionsstelle sollte sorgfältig gewählt und frei von entzündlichen Veränderungen sein. Eine Ausnahme stellen diagnostische oder therapeutische Punktionen bei bereits vorliegender Infektion dar (z. B. Entleerung eines eitrigen Ergusses, Abszesspunktion). Das 
Areal ist so weit freizulegen, dass eine Kontamination durch die Kleidung des Patienten sicher vermieden wird.

Hautantiseptik: Unmittelbar vor der Punktion ist eine Hautantiseptik unter Beachtung der vom Hersteller angegebenen (Mindest-)Einwirkzeit des Hautantiseptikums vorzunehmen. Zur Frage, ob das Hautantiseptikum durch Sprühen oder Wischen mit einem Tupfer aufgebracht werden soll, existieren nur wenige vergleichende Studien. Diese zeigen hinsichtlich der lokalen Desinfektionswirkung keinen Vorteil einer Wischdesinfektion; Studien mit klinischem Endpunkt existieren nicht [40, 41]. Das Hautantiseptikum kann daher aufgesprüht oder mittels Tupfer appliziert werden. Wenn ein Tupfer verwendet wird, richtet sich die Auswahl (keimarm oder steril) nach der mit der Punktion verbundenen Infektionsgefahr und ist im Rahmen der Risikoabschätzung (s. u.) im Hygieneplan punktionsspezifisch zu beschreiben.

Bei der Schleimhautantiseptik werden in der Humanmedizin fachspezifisch ggf. abweichende Verfahrensweisen beschrieben. (Siehe z. B. für die Zahnheilkunde: Empfehlung der Kommission für Krankenhaushygiene und Infektionsprävention „Infektionsprävention in der Zahnheilkunde - Anforderungen an die Hygiene“ [42]. Ein anderes Beispiel: Die Deutsche Ophtalmologische Gesellschaft empfiehlt bei der intravitrealen Injektion eine chirurgische (statt einer hygienischen) Händedesinfektion und das Schleimhautdesinfektionsmittel wird durch Tropfen in den Konjunktivalsack eingebracht [43].)

Barrieremaßnahmen beim Durchführenden und ggf. bei der/den Assistenzperson(en) sollen sicherstellen, dass ein exogener Keimeintrag in das Punktionsgebiet verhindert wird.

In der Literatur existieren nur wenige Studien, die wissenschaftlich begründete Aussagen zum Stellenwert einzelner Barrieremaßnahmen (z. B. Tragen von keimarmen oder sterilen Handschuhen, Schutzkittel, Mund-Nasen-Schutz) ermöglichen. Im Sinne einer Risikominimierung ist zu fordern, dass von der Kleidung der Durchführenden, sofern sie mit dem Patienten in Kontakt kommen kann, keine Kontaminationsgefahr ausgehen darf. Kohortenstudien und Berichte von Infektionszwischenfällen begründen das Tragen eines Mund-Nasen-Schutzes bei Anlage eines Periduralkatheters [44, 45, 46, 47]. Durch Analogieschluss können ferner die Ergebnisse von Studien, welche einen infektionspräventiven Effekt von maximalen sterilen Barrieremaßnahmen (d. h. Kopfhaube, Mund-Nasen-Schutz, langärmeliger steriler Bündchenkittel, sterile Handschuhe, großes Lochtuch oder Abdecktuch) bei Anlage von zentralen Gefäßkathetern gezeigt haben, auf andere langliegende perkutane Katheter übertragen werden $[48,49]$ (s. auch Empfehlung „Prävention Gefäßkatheter-assoziierter Infektionen").

Hygieneplan: Die Maßnahmen zur Desinfektion der Punktionsstelle und die persönlichen Schutzmaßnahmen des (der) Durchführenden müssen in einem einrichtungsspezifischen Hygieneplan festgelegt werden. Dabei sollte das methodenspezifische Risiko verschiedener Punktionsarten entsprechend den nachfolgend aufgeführten Risikogruppen von Punktionen berücksichtigt werden (vgl. Tab. 1).

\section{Risikogruppe 1:}

- Einfacher Punktionsablauf und

- geringes Risiko einer punktionsassoziierten Infektion

\section{Risikogruppe 2:}

- Einfacher Punktionsablauf und

- geringe Infektionsgefahr, aber in der Literatur dokumentierte schwerwiegende Infektionsfolgen beim (seltenen) Eintritt einer Infektion und

- keine Notwendigkeit der zwischenzeitlichen Ablage von sterilem Punktionszubehör.

\section{Risikogruppe 3:}

- Punktion von Organen oder Hohlräumen

oder

- komplexer Punktionsablauf mit Notwendigkeit der zwischenzeitlichen Ablage von sterilem Punktionszubehör, mit oder ohne Assistenzperson.

\section{Risikogruppe 4:}

- komplexe Punktion mit Notwendigkeit der zwischenzeitlichen Ablage von sterilem Punktionszubehör und steriler Anreichungen durch eine Assistenzperson und/oder

- Einbringung von Kathetern bzw. Fremdmaterial in Körperhöhlen oder tiefe Gewebsräume (z. B. Ventrikelkatheter, Periduralkatheter).

Die Zuordnung einzelner Punktionsarten zu diesen Risikogruppen ist nicht immer eindeutig möglich und bedarf im Einzelfall der Einschätzung der Hygienekommission (oder der sonst Verantwortlichen) vor Ort. Ein Beispiel für die Kernaussagen in einem solchen, einrichtungsspezifischen Hygieneplan gibt Tabelle 2.

Zusätzlich zu den im Hygieneplan beschriebenen Hygienemaßnahmen, welche sich am methodenspezifischen Risiko der Punktion orientieren, sind im Einzelfall stets auch individuelle, zusätzliche Risiken wie eine geschwächte Infektabwehr des $\mathrm{Pa}$ tienten (z. B. Immunsuppression) oder die Infektionsabwehr unterdrückende Eigenschaften des applizierten Medikamentes (z. B. Corticosteroide) zu berücksichtigen.

\section{Empfehlungen:}

- Die Maßnahmen zur Desinfektion der Punktionsstelle und die Barrieremaßnahmen des (der) Durchführenden müssen in einem einrichtungsspezifischen Hygieneplan festgelegt werden (Kat. IV).

- Das methodenspezifische Risiko verschiedener Punktionsarten sollte dabei entsprechend den Risikogruppen (vgl. Tabelle 2) berücksichtigt werden (Kat. II).

- Zusätzlich sind im Einzelfall individuelle Risiken des Patienten und/oder die Infektabwehr unterdrückende Eigenschaften des applizierten Medikamentes zu berücksichtigen (Kat. II).

- Das Personal ist regelmäßig anhand des Hygieneplans in den hygienischen Arbeitstechniken zu schulen (Kat. II). (Die Unterweisungen sind zu dokumentieren.) Der Erfolg der Schulungen sollte durch Beobachtung und Bewertung der Abläufe vor Ort, z. B. durch das Hygienefachpersonal, überprüft werden. 


\section{Empfehlung}

Tab. 2 Empfehlungen zur Berücksichtigung in einem Hygieneplan

\begin{tabular}{|c|c|c|c|c|c|}
\hline \multirow[b]{2}{*}{$\begin{array}{l}\text { Risiko- } \\
\text { gruppe }\end{array}$} & \multirow[b]{2}{*}{ Punktionsart } & \multirow[b]{2}{*}{$\begin{array}{l}\text { Tupferart } \\
\text { (s. Fußnote) }\end{array}$} & \multirow[b]{2}{*}{ Abdeckung } & \multicolumn{2}{|c|}{ Zusätzliche Schutzkleidung } \\
\hline & & & & $\begin{array}{l}\text { Durchführende } \\
\text { Person }\end{array}$ & Assistenz \\
\hline \multirow{6}{*}{$\begin{array}{l}\text { Risiko- } \\
\text { gruppe } 1\end{array}$} & i. c.-Injektion & keimarme & $\emptyset$ & nein & \multirow{6}{*}{$\begin{array}{l}\text { keine Assistenz } \\
\text { erforderlich }\end{array}$} \\
\hline & $\begin{array}{l}\text { s. c.-Injektion durch } \\
\text { med. Personal }\end{array}$ & keimarme & $\emptyset$ & nein & \\
\hline & Lanzettenblutentnahme & keimarme & $\emptyset$ & keimarme Handschuhe & \\
\hline & Blutabnahme & keimarme & $\emptyset$ & keimarme Handschuhe & \\
\hline & i. v.-Injektion (peripher) & keimarme & $\emptyset$ & keimarme Handschuhe & \\
\hline & $\begin{array}{l}\text { i.m.-Injektion (z. B. Schutz- } \\
\text { impfung) }\end{array}$ & keimarme & $\emptyset$ & nein & \\
\hline \multirow[t]{8}{*}{$\begin{array}{l}\text { Risiko- } \\
\text { gruppe } 2\end{array}$} & $\begin{array}{l}\text { S. C. -Punktion mit nachfolgender } \\
\text { Dauerapplikation }\end{array}$ & sterile & $\emptyset$ & keimarme Handschuhe & \multirow[t]{4}{*}{$\begin{array}{l}\text { keine Assistenz } \\
\text { erforderlich }\end{array}$} \\
\hline & $\begin{array}{l}\text { i. m.-Injektion (Risikopatient, } \\
\text { Injektion von Corticoiden oder } \\
\text { gewebstoxischen Substanzen) }\end{array}$ & sterile & $\emptyset$ & keimarme Handschuhe & \\
\hline & $\begin{array}{l}\text { Shunt-Punktion zur Dialyse } \\
\text { (autologer Shunt) }\end{array}$ & sterile & $\emptyset$ & keimarme Handschuhe & \\
\hline & Punktion einer Portkammer & sterile & $\emptyset$ & sterile Handschuhe & \\
\hline & Lumbalpunktion (diagnostisch) & sterile & $\begin{array}{l}\text { steriles Abdeck- oder } \\
\text { Lochtuch }\end{array}$ & sterile Handschuhe & \multirow{4}{*}{$\begin{array}{l}\text { keine besonderen } \\
\text { Anforderungen an } \\
\text { die Assistenz }\end{array}$} \\
\hline & $\begin{array}{l}\text { Punktion eines Ommaya- oder } \\
\text { Rickham-Reservoirs }\end{array}$ & sterile & $\emptyset$ & $\begin{array}{l}\text { - sterile Handschuhe } \\
\text { - Mund-Nasen-Schutz } \\
\text { bei Punktion mit } \\
\text { Spritzenwechsel }\end{array}$ & \\
\hline & Blasenpunktion (diagnostisch) & sterile & $\emptyset$ & sterile Handschuhe & \\
\hline & $\begin{array}{l}\text { Pleurapunktion, Ascitespunktion } \\
\text { (diagnostisch) }\end{array}$ & sterile & $\emptyset$ & $\begin{array}{l}\text { - sterile Handschuhe } \\
\text { - Mund-Nasen-Schutz }\end{array}$ & \\
\hline \multirow[t]{8}{*}{$\begin{array}{l}\text { Risiko- } \\
\text { gruppe } 3\end{array}$} & Beckenkammpunktion & sterile & $\begin{array}{l}\text { steriles Abdeck-oder } \\
\text { Lochtuch }\end{array}$ & sterile Handschuhe & \multirow{5}{*}{$\begin{array}{l}\text { keine besonderen } \\
\text { Anforderungen an } \\
\text { die Assistenz }\end{array}$} \\
\hline & $\begin{array}{l}\text { Amniozentese } \\
\text { Chorionzottenbiopsie }\end{array}$ & sterile & $\begin{array}{l}\text { steriles Abdeck- oder } \\
\text { Lochtuch }\end{array}$ & sterile Handschuhe & \\
\hline & $\begin{array}{l}\text { Transvaginale (schallkopf- } \\
\text { gesteuerte) Zysten- oder } \\
\text { Gewebspunktion }\end{array}$ & sterile & $\begin{array}{l}\text { steriles Abdeck- oder } \\
\text { Lochtuch }\end{array}$ & sterile Handschuhe & \\
\hline & $\begin{array}{l}\text { Organpunktion (z. B. Niere, } \\
\text { Leber, Lymphknoten, Milz, } \\
\text { Schilddrüse) }\end{array}$ & sterile & $\begin{array}{l}\text { steriles Abdeck- oder } \\
\text { Lochtuch }\end{array}$ & sterile Handschuhe & \\
\hline & $\begin{array}{l}\text { Anlage einer suprapubischen } \\
\text { Ableitung }\end{array}$ & sterile & $\begin{array}{l}\text { steriles Abdeck- oder } \\
\text { Lochtuch }\end{array}$ & $\begin{array}{l}\text { - sterile Handschuhe } \\
\text { - Mund-Nasen-Schutz }\end{array}$ & \\
\hline & $\begin{array}{l}\text { Spinalanästhesie (Single shot), } \\
\text { intrathekale Medikamenten- } \\
\text { applikation }\end{array}$ & sterile & $\begin{array}{l}\text { steriles Abdeck- oder } \\
\text { Lochtuch }\end{array}$ & $\begin{array}{l}\text { - sterile Handschuhe } \\
\text { - Mund-Nasen-Schutz }\end{array}$ & Mund-Nasen-Schutz \\
\hline & $\begin{array}{l}\text { Gelenkpunktion (diagnostisch } \\
\text { bzw. mit Einzelinjektion)* }\end{array}$ & sterile & $\begin{array}{l}\text { steriles Abdeck- oder } \\
\text { Lochtuch }\end{array}$ & $\begin{array}{l}\text { - sterile Handschuhe } \\
\text { - Mund-Nasen-Schutz } \\
\text { bei Punktion mit } \\
\text { Spritzenwechsel }\end{array}$ & \multirow[t]{2}{*}{$\begin{array}{l}\text { Mund-Nasen-Schutz } \\
\text { bei Punktion mit } \\
\text { Spritzenwechsel }\end{array}$} \\
\hline & $\begin{array}{l}\text { Vorderkammerpunktion des } \\
\text { Auges mit intravitrealer Medika- } \\
\text { mentengabe }\end{array}$ & sterile & $\begin{array}{l}\text { steriles Abdeck- oder } \\
\text { Lochtuch }\end{array}$ & $\begin{array}{l}\text { - sterile Handschuhe } \\
\text { - Mund-Nasen-Schutz } \\
\text { bei Punktion mit } \\
\text { Spritzenwechsel }\end{array}$ & \\
\hline
\end{tabular}




\begin{tabular}{|c|c|c|c|c|c|}
\hline \multirow[b]{2}{*}{$\begin{array}{l}\text { Risiko- } \\
\text { gruppe }\end{array}$} & \multirow[b]{2}{*}{ Punktionsart } & \multirow[b]{2}{*}{$\begin{array}{l}\text { Tupferart } \\
\text { (s. Fußnote) }\end{array}$} & \multirow[b]{2}{*}{ Abdeckung } & \multicolumn{2}{|c|}{ Zusätzliche Schutzkleidung } \\
\hline & & & & $\begin{array}{l}\text { Durchführende } \\
\text { Person }\end{array}$ & Assistenz \\
\hline \multirow[t]{3}{*}{$\begin{array}{l}\text { Risiko- } \\
\text { gruppe } 4\end{array}$} & $\begin{array}{l}\text { Anlage einer Bülau-Drainage, } \\
\text { eines Pleuracath, } \\
\text { einer Monaldi-Drainage }\end{array}$ & sterile & $\begin{array}{l}\text { steriles Abdeck- oder } \\
\text { Lochtuch }\end{array}$ & $\begin{array}{l}\text { - Mund-Nasen-Schutz } \\
\text { - OP-Haube } \\
\text { - steriler langärmeliger } \\
\text { Kittel } \\
\text { - sterile Handschuhe }\end{array}$ & Mund-Nasen-Schutz \\
\hline & $\begin{array}{l}\text { Periduralanästhesie/Spinalan- } \\
\text { ästhesie mit Katheteranlage, } \\
\text { Anlage eines Periduralkatheters } \\
\text { zur Schmerztherapie }\end{array}$ & sterile & $\begin{array}{l}\text { steriles Abdeck- oder } \\
\text { Lochtuch }\end{array}$ & $\begin{array}{l}\text { - Mund-Nasen-Schutz } \\
\text { - OP-Haube } \\
\text { - sterile Handschuhe } \\
\text { - steriler langärmeliger } \\
\text { Kittel }\end{array}$ & $\begin{array}{l}\text { - unsterile Handschuhe } \\
\text { - Mund-Nasen-Schutz }\end{array}$ \\
\hline & $\begin{array}{l}\text { Perkutane endoskopische } \\
\text { Gastrostomie-Anlage (PEG) }\end{array}$ & sterile & $\begin{array}{l}\text { steriles Abdeck- oder } \\
\text { Lochtuch }\end{array}$ & $\begin{array}{l}\text { - sterile Handschuhe } \\
\text { - OP-Haube } \\
\text { - steriler langärmeliger } \\
\text { Kittel } \\
\text { - Mund-Nasen-Schutz }\end{array}$ & $\begin{array}{l}\text { - unsterile Handschuhe } \\
\text { - Mund-Nasen-Schutz } \\
\text { - ggf. Einwegschürze }\end{array}$ \\
\hline \multicolumn{6}{|c|}{$\begin{array}{l}\text { Anmerkungen: } \\
\text { Vor allen aufgeführten Punktionen, ggf. auch bei Zwischenschritten, ist eine hygienische Händedesinfektion erforderlich. } \\
\text { Zur Hautantisepsis. Bei allen Punktionen kann die Hautantisepsis grundsätzlich auch durch alleiniges Einsprühen erfolgen. Sollen Tupfer verwendet werden, so empfehlen } \\
\text { sich die angegebenen Tupferqualitäten. Die vom Hersteller angegebene Einwirkzeit des Hautantiseptikums ist bei beiden Verfahrensweisen zu beachten. Vor der Punktion } \\
\text { muss das Hautantiseptikum abgetrocknet sein. Bei Punktionen, bei denen keine spezielle Einkleidung angegeben ist, wird das Tragen kurzärmeliger Kleidung empfohlen. } \\
\emptyset=\text { nicht erforderlich } \\
\text { * = Deutsche Gesellschaft für Orthopädie und Orthopädische Chirurgie (2008) "Hygienemaßnahmen bei intraartikulären Punktionen und Injektionen", } \\
\text { AWMF-Leitlinie Nr. 029/006. www.awmf.de }\end{array}$} \\
\hline
\end{tabular}

\section{Spezielle Hygiene- und Prophylaxemaßnahmen bei Insertion nicht-intravasaler Katheter}

Für Punktionen, die der Insertion nichtintravasaler Katheter mit geplanter längerer Liegedauer dienen, existieren nur wenige wissenschaftliche Daten, die die Einhaltung bestimmter hygienischer Mindestanforderungen begründen. Dabei handelt es sich nicht um randomisierte Studien mit klinischem Endpunkt.

In Form von Fallberichten oder Fallserien wurde über Meningitiden oder peridurale Abszesse nach der Anlage von rückenmarksnahen Kathetern berichtet, die meist durch Erreger der Haut- (z. B. Staphylococcus aureus) oder Rachenflora (z. B. Streptococcus salivarius) verursacht wurden $[44,45,46,47,50]$. In Einzelfällen konnte der Nasen-Rachen-Raum des durchführenden Arztes aufgrund molekularer Typisierung der Erreger als Infektionsquelle identifiziert werden [47]. Diese Beobachtungen begründen die Forderung nach generellem Tragen eines MundNasen-Schutzes bei Anlage eines Peridural- oder Spinalkatheters zur perioperativen Analgesie oder Schmerztherapie [46,
51]. Analog ist dies auch bei Einzelinjektionen in den Spinalraum, die einen Spritzenwechsel auf der Lumbalpunktionskanüle erfordern, notwendig. Für andere Katheteranlagen (z. B. Anlage einer externen Liquordrainage, Bülau-Drainage, Ascitesdrainage, peritonealen Katheteranlage für die kontinuierliche Peritonealdialyse u. a.) existieren keine wissenschaftlichen Daten zum Umfang der notwendigen Hygienemaßnahmen. Katheteranlagen, die Hautuntertunnelungen erfordern, werden üblicherweise als Operation in einem OP-Saal durchgeführt.

Für bestimmte Punktions- bzw. Katheterarten liegen randomisierte klinische Studien zum Einfluss einer Antibiotikaprophylaxe vor. Der Effekt einer Singleshot-Antibiotikaprophylaxe wurde für die Anlage perkutaner endoskopischer Gastrostomien (PEG) untersucht [52, 53, 54, 55]. Die Studien bestätigten ausnahmslos einen signifikanten Effekt im Hinblick auf Infektionen des Stichkanals; eine Single-shot-Antibiotikaprophylaxe wird daher in der Literatur empfohlen $[56,57]$. Bei der chirurgischen Anlage von getunnelten, dauerhaft implantierten Kathetern zur kontinuierlichen Peritonealdialyse (Tenckhoff-Katheter) zeigen Studien einen positiven Effekt einer intravenösen Antibiotikaprophylaxe in Bezug auf die Peritonitisrate $[58,59]$. Studien zur Wertigkeit einer zusätzlichen intraperitonealen Prophylaxe über die Dialyseflüssigkeit fehlen. Im Analogieschluss sollte bei der (stationsbasierten) Anlage eines Katheters zur kurzfristigen Peritonealdialyse mittels Stilett- oder Seldinger-Technik eine intravenöse Single-shot-Prophylaxe durchgeführt werden $[60,61,62]$.

Bei der Anlage von externen Ventrikelkathetern wird von der Mehrzahl der praktizierenden Neurochirurgen eine Antibiotikaprophylaxe durchgeführt [22, 63]. Eine Cochrane-Analyse randomisierter und quasi-randomisierter Studien zeigte einen Vorteil einer über 24 Stunden nach dem Eingriff gegebenen i.v.-Antibiotikaprophylaxe in Bezug auf Shunt-assoziierte Infektionen. Ob eine länger dauernde Prophylaxe darüber hinaus von Vorteil ist, konnte aufgrund der Studiendaten nicht entschieden werden [64].

Für externe Ventrikelableitungen sind silberimprägnierte bzw. antibiotikabeschichtete Katheter verfügbar, deren antimikrobieller Effekt in vitro nachgewiesen wurde [65]. Klinische Einschätzungen und Studiendaten zu diesen Kathetern 
sind überwiegend positiv, weitere Untersuchungen hierzu sind jedoch erforderlich $[64,66,67,68,69]$.

\section{Empfehlungen:}

- Bei Anlage eines Peridural- oder Spinalkatheters oder bei einer Einzelinjektion in den Spinalkanal ist ein Mund-NasenSchutz anzulegen (Kat. II).

- Bei Punktionen, die der Anlage von perkutanen Kathetern in Körperhöhlen oder tiefe Gewebsräume dienen (vgl. Tabelle 2, Punktionen der Risikogruppe 4), sind maximale hygienische Barrieremaßnahmen einzuhalten (Einkleidung des Durchführenden mit Kopfhaube, Mund-Nasen-Schutz, langärmeligem sterilen Bündchenkittel und sterilen Handschuhen, Verwendung eines großen sterilen Abdeck- oder Lochtuchs) (Kat. IB).

- Bezüglich einer Antibiotikaprophylaxe bei bestimmten Punktionsarten wird auf Empfehlungen der Fachgesellschaften verwiesen [70].

- Die Verwendung antimikrobieller Ventrikelkatheter zur kontinuierlichen externen Liquorableitung soll im Einzelfall entschieden werden (Kat. II).

\section{Punktionen unter Ultraschallkontrolle}

Zusätzlich zu der gebotenen Desinfektion von Ultraschallköpfen zwischen zwei Patienten ist bei ultraschallgeführten Punktionen ein steriler Überzug aufzuziehen, wenn der Schallkopf die Punktionsstelle direkt berührt oder während der Punktion mit der Nadel in Kontakt kommt. Letzteres ist zum Beispiel auch der Fall, wenn die Nadel direkt durch einen speziellen Punktionsschallkopf geführt wird. Handelt es sich um Punktionen und Injektionen ohne Anlage eines Katheters, reicht die sterile Ummantelung nur des Ultraschallkopfes aus. Wird ein Katheter gelegt, müssen Schallkopf und Zuleitungskabel eine sterile Ummantelung erhalten. Wird unsteriles Schallleitungsmedium verwendet, darf es hierdurch nicht zur Kontamination der Nadel oder des Punktionsgebietes kommen. Dies ist z. B. gewährleistet, wenn der Schallkopf an einer separaten Stelle entfernt vom Punktionsgebiet aufgesetzt wird. Bei Verstößen gegen diesen
Grundsatz sind Infektionszwischenfälle vorgekommen [71]. Ist das Auftragen von Schallleitungsmedium direkt an der Punktionsstelle unverzichtbar, so kann entweder alkoholisches Hautdesinfektionsmittel oder spezielles, steriles Ultraschallgel verwendet werden $[72,73,74]$.

Die nachfolgenden Empfehlungen können nicht mit einer Kategorie versehen werden, da keine randomisierten Studien mit klinischem Endpunkt und nur vereinzelt hinweisende wissenschaftliche Daten vorliegen. Die Empfehlungen basieren auf dem Gebot der Risikominimierung bei einem voll beherrschbaren Risiko.

\section{Empfehlungen:}

- Bei ultraschallgeführten Punktionen, bei denen der Schallkopf die Punktionsstelle berührt oder mit der Punktionsnadel in Kontakt kommen kann, ist der Schallkopf mit einem sterilen Überzug zu versehen.

- Bei ultraschallgeführten Punktionen, die der Insertion eines Katheters dienen, muss die sterile Ummantelung auch das Zuleitungskabel umfassen.

- Wird unsteriles Schallleitungsmedium verwendet, darf es hierdurch nicht zur Kontamination der Nadel oder des Punktionsgebietes kommen.

- Wird Schallleitungsmedium direkt an der Punktionsstelle benötigt, ist alkoholisches Hautdesinfektionsmittel oder steriles Ultraschallgel zu verwenden.

\section{Versorgung der Punktionsstelle nach Beendigung des Eingriffs}

Nach Punktionen der Risikogruppen 1 und 2 kann die Punktionsstelle mit einem keimarmen Wundschnellverband (Pflaster) versorgt werden. Wurden Organe oder Körperhöhlen punktiert, ist die Punktionsstelle zumindest mit einem sterilen Pflaster zu versorgen. Der Patient ist über postpunktionelle Risiken (z. B. mögliche Infektionszeichen wie Schwellung, Schmerzen, Rötung, Fieber) angemessen $\mathrm{zu}$ informieren und soll entsprechende Verhaltenshinweise (z. B. durch Aushändigung eines Merkblattes) erhalten.
11 Ergänzende Hinweise für Punktionen und Injektionen bei Diabetes mellitus

Pen-Geräte sind stets patientenbezogen zu verwenden. Geräte zur Blutglukosemessung und Lanzettblutentnahme-Geräte sind entsprechend den Herstellerinformationen $\mathrm{zu}$ verwenden. Werden im klinischen Alltag Blutglukosemessungen bei verschiedenen Patienten hintereinander durchgeführt, ist die Verwendung von Einmallanzetten die beste Lösung. Vor Lanzettblutentnahmen und Insulininjektionen ist ebenso wie bei jeder anderen Punktion, die durch medizinisches Personal durchgeführt wird, eine Hautdesinfektion durchzuführen. Bei jeder Insulininjektion durch medizinisches Personal ist eine frische Nadel zu verwenden (www. rki.de $>$ Infektionsschutz $>$ Krankenhaushygiene $>$ FAQ $>$ Insulingabe; [75]). Hinsichtlich der Eigendurchführung von Lanzettblutentnahmen und Insulininjektionen durch den Patienten selbst im häuslichen Umfeld wird auf Leitlinien der Fachgesellschaften verwiesen [2].

Die Empfehlungen wurden ehrenamtlich und ohne Einflussnahme kommerzieller Interessengruppen im Auftrag der Kommission für Krankenhaushygiene und Infektionsprävention bearbeitet von Prof. Dr. med. Matthias Trautmann (Leiter der Arbeitsgruppe), Stuttgart; PD Dr. med. Martin Bald, Stuttgart; Prof. Dr. med. Axel Kramer, Greifswald; Prof. Dr. med. Joachim Martius, Agatharied; Frau Erika Voggesberger, Regensburg; Prof. Dr. med. Manfred Weiss, Ulm; Dr. med. Nicoletta Wischnewski, Berlin und Dr. med. Alfred Nassauer (für das RKI).

\section{Literatur}

1. Kommission für Krankenhaushygiene und Infektionsprävention (2010) Die Kategorien in der Richtlinie für Krankenhaushygiene und Infektionsprävention - Aktualisierung der Definitionen Bundesgesundheitsbl 53:754-756

2. Martin S, Dreher M, Kiess W (2008) Behandlung des Diabetes mellitus Typ 1. In: Scherbaum WA: Praxis-Leitlinien der Deutschen Diabetes-Gesellschaft, Elsevier Verlag 2:1162-1167.

3. Trautmann M (2006) Hautdesinfektion vor der Insulinapplikation. Hygiene und Medizin 31:239-240

4. Komission für Krankenhaushygiene und Infektionsprävention (2000) Händehygiene. Bundesgesundheitsbl 43:230-233 
5. Kommission für Krankenhaushygiene und Infektionsprävention (2002) Prävention Gefäßkatheter-assoziierter Infektionen. Bundesgesundheitsbl 45:907-924

6. Komission für Krankenhaushygiene und Infektionsprävention (2004) Anforderungen an die Hygiene bei der Reinigung und Desinfektion von Flächen. Bundesgesundheitsbl 47:51-61

7. Kommission für Krankenhaushygiene und Infektionsprävention (2007) Prävention postoperativer Infektionen im OP-Gebiet. Bundesgesundheitsbl 50:377-393

8. Kommission für Krankenhaushygiene und Infektionsprävention (2000) Anforderungen der Hygiene bei Operationen und anderen invasiven Eingriffen. Bundesgesundheitsbl 43:644-648

9. Komission für Krankenhaushygiene und Infektionsprävention (2007) Anforderungen der Krankenhaushygiene und des Arbeitsschutzes an die Hygienebekleidung und persönliche Schutzausrüstung. Epidemiol Bulletin 1:3-4

10. Kommission für Krankenhaushygiene und Infektionsprävention (2009) Personelle und organisatorische Voraussetzungen zur Prävention nosokomialer Infektionen. Bundesgesundheitsbl 52:951-962

11. Komission für Krankenhaushygiene und Infektionsprävention (2010) Anforderungen an die Hygiene bei der medizinischen Versorgung von immunsupprimierten Patienten. Bundesgesundheitsbl 53:357-388

12. Arbeitsgemeinschaft der Obersten Landesgesundheitsbehörden (AOLG) (1998) Herstellung applikationsfertiger Zytostatikalösungen in Apotheken. Bundesgesundheitsbl 41:404-409

13. Arbeitskreis deutscher Krankenhausapotheker (2003) Aseptische Herstellung und Prüfung applikationsfertiger Parenteralia. Krankenhauspharmazie 24:195-201

14. Bundesapothekenkammer (2008) Leitlinie der Bundesapothekerkammer zur Qualitätssicherung bei der Herstellung und Prüfung applikationsfertiger Parenteralia ohne toxisches Potential. http://www. abda.de

15. Clemens S, Kramer A, Reese M, Schwesinger G, Rudolph P (1998) Keimverschleppung und Stanzzylinder bei unterschiedlichen Injektionskanülen. In: Ohrloff C, Kohnen T, Duncker G. 11. Kongress der Deutschen Gesellschaft für Intraokularlinsen- Implantation und refraktive Chirugie. Springer-Verlag New York Berlin Heidelberg: 423-431

16. Heeg P, Bernau A, Dauber W (1990) Infektionsgefahr durch Injektionen. Biomed Technik 35:179-181

17. Austin PD, Elia M (2009) A systematic review and meta-analysis of the risk of microbial contamination of aseptically prepared doses in different environments. J Pharm Pharm Sci 12:233-242

18. Charalambous CP, Tryfonidis M, Sadiq S, Hirst P, Paul A (2003) Septic arthritis following intra-articular steroid injection of the knee--a survey of current practice regarding antiseptic technique used during intra-articular steroid injection of the knee. Clin Rheumatol 22:386-390

19. Frick S, Cerny A (2001) Necrotizing fasciitis due to Streptococcus pneumoniae after intramuscular injection of nonsteroidal anti-inflammatory drugs: report of 2 cases and review. Clin Infect Dis 33:740744

20. Lehnhardt M, Vu P, Kuhnen C (2005) Fatale Folgen von Injektionen. Eine Analyse zur Häufigkeit, Komplikationsmanagement, Prophylaxe und ökonomischen Aspekten. Zentralbl Chir 130:162-169
21. Zopf Y, Konturek P, Nuernberger A, Maiss J, Zenk J, Iro H, Hahn EG, Schwab D (2008) Local infection after placement of percutaneous endoscopic gastrostomy tubes: a prospective study evaluating risk factors. Can J Gastroenterol 22:987-991

22. Poon WS, Ng S, Wai S (1998) CSF antibiotic prophylaxis for neurosurgical patients with ventriculostomy: a randomised study. Acta Neurochir Suppl 71:146-148

23. Archibald LK, Ramos M, Arduino MJ, Aguero SM, Deseda C, Banerjee S, Jarvis WR (1998) Enterobacter cloacae and Pseudomonas aeruginosa polymicrobial bloodstream infections traced to extrinsic contamination of a dextrose multidose vial. J Pediatr 133:640-644

24. Bruguera M, Saiz JC, Franco S, Gimenez-Barcons M, Sanchez-Tapias JM, Fabregas S, Vega R, Camps N, Dominguez A, Salleras L (2002) Outbreak of nosocomial hepatitis $C$ virus infection resolved by genetic analysis of HCV RNA. J Clin Microbiol 40:43634366

25. Dolan SA, Felizardo G, Barnes S, Cox TR, Patrick M, Ward KS, Arias KM (2010) APIC position paper: safe injection, infusion, and medication vial practices in health care. Am J Infect Control 38:167-172

26. Grohskopf LA, Roth VR, Feikin DR, Arduino MJ, Carson LA, Tokars Jl, Holt SC, Jensen BJ, Hoffman RE, Jarvis WR (2001) Serratia liquefaciens bloodstream infections from contamination of epoetin alfa at a hemodialysis center. N Engl J Med 344:1491-1497

27. Katzenstein TL, Jorgensen LB, Permin H, Hansen J, Nielsen C, Machuca R, Gerstoft J (1999) Nosocomial HIV-transmission in an outpatient clinic detected by epidemiological and phylogenetic analyses. AIDS 13:1737-1744

28. Kokubo S, Horii T, Yonekawa O, Ozawa N, Mukaide M (2002) A phylogenetic-tree analysis elucidating nosocomial transmission of hepatitis $C$ virus in a haemodialysis unit. J Viral Hepat 9:450-454

29. Murray RJ, Pearson JC, Coombs GW, Flexman JP, Golledge CL, Speers DJ, Dyer JR, McLellan DG, Reilly M, Bell JM, Bowen SF, Christiansen KJ (2008) Outbreak of invasive methicillin-resistant Staphylococcus aureus infection associated with acupuncture and joint injection. Infect Control Hosp Epidemiol 29:859-865

30. Oren I, Hershow RC, Ben-Porath E, Krivoy N, Goldstein N, Rishpon S, Shouval D, Hadler SC, Alter MJ, Maynard JE, et al. (1989) A common-source outbreak of fulminant hepatitis B in a hospital. Ann Intern Med 110:691-698

31. Petrosillo N, Ippolito G, Solforosi L, Varaldo PE, Clementi M, Manzin A (2000) Molecular epidemiology of an outbreak of fulminant hepatitis B. J Clin Microbiol 38:2975-2981

32. Simon PA, Chen RT, Elliott JA, Schwartz B (1993) Outbreak of pyogenic abscesses after diphtheria and tetanus toxoids and pertussis vaccination. Pediatr Infect Dis J 12:368-371

33. Hennequin C, Kauffmann-Lacroix C, Jobert A, Viard JP, Ricour C, Jacquemin JL, Berche P (2000) Possible role of catheters in Saccharomyces boulardii fungemia. Eur J Clin Microbiol Infect Dis 19:16-20

34. Hota S, Hirji Z, Stockton K, Lemieux C, Dedier H, Wolfaardt G, Gardam MA (2009) Outbreak of multidrug-resistant Pseudomonas aeruginosa colonization and infection secondary to imperfect intensive care unit room design. Infect Control Hosp Epidemiol 30:25-33

35. Sissoko B, Sütterlin R, Blaschke M, Stefaniak S, Daeschlein G, Kramer A (2005) Emission von Bakterien aus Geruchsverschlüssen. Hygiene und Medizin 30:100-104
36. Schulze-Röbbecke R (2009) Standardmaßnahmen zur Prävention der Übertragung nosokomialer Infektionen - Standardhygiene, Basishygiene. Krankenhaushygiene up2date 4:193-207

37. Krause G, Trepka MJ, Whisenhunt RS, Katz D, Nainan O, Wiersma ST, Hopkins RS (2003) Nosocomial transmission of hepatitis $C$ virus associated with the use of multidose saline vials. Infect Control Hosp Epidemiol 24:122-127

38. Garcia-Caballero J, Herruzo-Cabrera H, Vera-Cortes ML, Garcia de Lorenzo A, Vazquez-Encinar A, Garcia-Caballero F, del Rey-Calero J (1985) The growth of micro-organisms in intravenous fluids. J Hosp Infect 6:154-157

39. Hugbo PG, Imhanlahimi WA (1983) Growth of bacteria in intravenous fluids under stimulated actualuse conditions. Am J Hosp Pharm 40:998-1001

40. Debreceni G, Meggyesi R, Mestyan G (2007) Efficacy of spray disinfection with a 2-propanol and benzalkonium chloride containing solution before epidural catheter insertion--a prospective, randomized, clinical trial. Br J Anaesth 98:131-135

41. Heeg P, Bernau A (1984) Vergleichende Untersuchungen zur Prüfung der Wirksamkeit verschiedener Hautdesinfektionsverfahren. Hyg Med 9:468470

42. Kommission für Krankenhaushygiene und Infektionsprävention (2006) Infektionsprävention in der Zahnheilkunde - Anforderungen an die Hygiene. Bundesgesundheitsbl 49:375-394

43. Jaisslie G, Bartz-Schmidt K (April 2007) Empfehlung der Deutschen Ophthalmologischen Gesellschaft, der Retinologischen Gesellschaft und des Berufsverbandes der Augenärzte Deutschlands für die Durchführung von intravitrealen Injektionen (IV). www.augeninfo.de

44. Holt HM, Andersen SS, Andersen O, Gahrn-Hansen B, Siboni K (1995) Infections following epidural catheterization. J Hosp Infect 30:253-260

45. Kindler CH, Seeberger MD, Staender SE (1998) Epidural abscess complicating epidural anesthesia and analgesia. An analysis of the literature. Acta Anaesthesiol Scand 42:614-620

46. Rubin L, Sprecher H, Kabaha A, Weber G, Teitler N, Rishpon S (2007) Meningitis following spinal anesthesia: 6 cases in 5 years. Infect Control Hosp Epidemiol 28:1187-1190

47. Trautmann M, Lepper PM, Schmitz FJ (2002) Three cases of bacterial meningitis after spinal and epidural anesthesia. Eur J Clin Microbiol Infect Dis 21:43-45

48. Mermel LA, McCormick RD, Springman SR, Maki DG (1991) The pathogenesis and epidemiology of catheter-related infection with pulmonary artery Swan-Ganz catheters: a prospective study utilizing molecular subtyping. Am J Med 91:197S-205S

49. Raad II, Hohn DC, Gilbreath BJ, Suleiman N, Hill LA, Bruso PA, Marts K, Mansfield PF, Bodey GP (1994) Prevention of central venous catheter-related infections by using maximal sterile barrier precautions during insertion. Infect Control Hosp Epidemiol 15:231-238

50. Scott DB, Hibbard BM (1990) Serious non-fatal complications associated with extradural block in obstetric practice. Br J Anaesth 64:537-541

51. Morin A, Kerwat K, Büttner J (2010) Hygieneempfehlungen für die Anlage und weiterführende Versorgung von Regionalanästhesie-Verfahren: www. ak-regionalanaesthesie.dgai.de 
52. Dormann AJ, Wigginghaus B, Risius H, Kleimann F, Kloppenborg A, Grunewald T, Huchzermeyer $\mathrm{H}$ (1999) A single dose of ceftriaxone administered 30 minutes before percutaneous endoscopic gastrostomy significantly reduces local and systemic infective complications. Am J Gastroenterol 94:3220-3224

53. Jafri N, Mahid S, Minor K, Idstein S, Hornung C, Galandiuk S (2007) Meta-analysis: antibiotic prophylaxis to prevent peristomal infection following percuteanous endoscopic gastrostomy. Aliment Pharmacol Ther 25:647-656

54. Preclik G, Grune S, Leser HG, Lebherz J, Heldwein W, Machka K, Holstege A, Kern WV (1999) Prospective, randomised, double blind trial of prophylaxis with single dose of co-amoxiclav before percutaneous endoscopic gastrostomy. BMJ 319:881-884

55. Saadeddin A, Freshwater DA, Fisher NC, Jones BJ (2005) Antibiotic prophylaxis for percutaneous endoscopic gastrostomy for non-malignant conditions: a double-blind prospective randomized controlled trial. Aliment Pharmacol Ther 22:565-570

56. Lin HS, Ibrahim HZ, Kheng JW, Fee WE, Terris DJ (2001) Percutaneous endoscopic gastrostomy: strategies for prevention and management of complications. Laryngoscope 111:1847-1852

57. Lipp A, Lusardi G (2009) A systematic review of prophylactic antimicrobials in PEG placement. J Clin Nurs 18:938-948

58. Gadallah MF, Ramdeen G, Torres C, Mignone J, Patel D, Mitchell L, Tatro S (2000) Preoperative vancomycin prophylaxis for newly placed peritoneal dialysis catheters prevents postoperative peritonitis. Adv Perit Dial 16:199-203

59. Wikdahl AM, Engman U, Stegmayr BG, Sorenssen JG (1997) One-dose cefuroxime i.v. and i.p. reduces microbial growth in PD patients after catheter insertion. Nephrol Dial Transplant 12:157-160

60. Bonifati C, Pansini F, Torres DD, Navaneethan SD, Craig JC, Strippoli GF (2006) Antimicrobial agents and catheter-related interventions to prevent peritonitis in peritoneal dialysis: Using evidence in the context of clinical practice. Int J Artif Organs 29:41-49

61. Salman L, Asif A (2009) Antibiotic prophylaxis: is it needed for dialysis access procedures? Semin Dial 22:297-299

62. Strippoli G, Tong A , Johnson D, Schena FP (2004) Antimicrobial agents for preventing peritonitis in peritoneal dialysis patients. Cochrane Database Syst Rev 18:CD004679

63. McCarthy PJ, Patil S, Conrad SA, Scott LK (2010) International and specialty trends in the use of prophylactic antibiotics to prevent infectious complications after insertion of external ventricular drainage devices. Neurocrit Care 12:220-224

64. Ratilal B, Costa J, Sampaio C (2006) Antibiotic prophylaxis for surgical introduction of intracranial ventricular shunts. Cochrane Database of Systematic Reviews Art. No.: CD005365. DOI: 005310.001002/14651858.CD14005365. pub14651852

65. Secer HI, Kural C, Kaplan M, Kilic A, Duz B, Gonul E, Izci Y (2008) Comparison of the efficacies of antibiotic-impregnated and silver-impregnated ventricular catheters on the prevention of infections. An in vitro laboratory study. Pediatr Neurosurg 44:444-447

66. Fichtner J, Guresir E, Seifert V, Raabe A (2010) Efficacy of silver-bearing external ventricular drainage catheters: a retrospective analysis. J Neurosurg 112:840-846
67. Galiano K, Pleifer C, Engelhardt K, Brossner G, Lackner P, Huck C, Lass-Florl C, Obwegeser A (2008) Silver segregation and bacterial growth of intraventricular catheters impregnated with silver nanoparticles in cerebrospinal fluid drainages. Neurol Res 30:285-287

68. Lackner P, Beer R, Broessner G, Helbok R, Galiano K, Pleifer C, Pfausler B, Brenneis C, Huck C, Engelhardt K, Obwegeser AA, Schmutzhard E (2008) Efficacy of silver nanoparticles-impregnated external ventricular drain catheters in patients with acute occlusive hydrocephalus. Neurocrit Care 8:360-365

69. Zabramski JM, Whiting D, Darouiche RO, Horner TG, Olson J, Robertson C, Hamilton AJ (2003) Efficacy of antimicrobial-impregnated external ventricular drain catheters: a prospective, randomized, controlled trial. J Neurosurg 98:725-730

70. Wacha H, Hoyme U, Isenmann R, Kujath P, Lebert C, Naber K, Salzberger B (2010) Perioperative Antibiotikaprophylaxe. Empfehlung einer Expertenkommission der Paul-Ehrlich-Gesellschaft für Chemotherapie e.V. Chemotherapie Journal 19:70-84

71. Olshtain-Pops K, Block C, Temper V, Hidalgo-Grass C, Gross I, Moses AE, Gofrit ON, Benenson S (2011) An outbreak of achromobacter xylosoxidans associated with ultrasound gel used during transrectal ultrasound guided prostate biopsy. J Urol 185:144-147

72. Hajjar J, Mounier M (2009) Echographie en anésthesie et mesures de prévention du risque infectieux [letter]. Annales francaises d'Anesthésie et de Réanimation 28:397-398

73. Haut Conseil de la Santé Publique (2007) Comité technique des infections nosocomiales et des infections liées aux soins. Gaines de protection à usage unique pour dispositifs medicaux réutilisables: recommandations d'utilization. www.hcsp.fr

74. Hillmann R, Döffert J (2009) Hygieneempfehlungen für interventionelle Verfahren. Hillmann R, Döffert J. Praxis der anästhesiologischen Sonografie, Elsevier Verlag :31-33

75. Deutsche Gesellschaft für Krankenhaushygiene (2010) Konsensuspapier zur Mehrfachverwendung von Injektionskanülen bei Insulinpens und InsulinEinmalspritzen und zur Hautantiseptik (Hautdesinfektion) vor der subkutanen Insulininjektion. Hyg Med 35:380-381

76. Cooper CL, Gerstein R, Kaita K (2000) Fasciitis complicating subcutaneous injection of interferon beta. Can J Gastroenterol 14 Suppl B:81B-82B

77. Finucane K, Ambrey P, Narayan S, Archer CB, Dayan C (2003) Insulin injection abscesses caused by Mycobacterium chelonae. Diabetes Care 26:24832484

78. Lloyd-Smith E, Wood E, Zhang R, Tyndall MW, Montaner JS, Kerr T (2008) Risk factors for developing a cutaneous injection-related infection among injection drug users: a cohort study. BMC Public Health 8:405

79. Abbas SQ, Yeldham M, Bell S (2005) The use of metal or plastic needles in continuous subcutaneous infusion in a hospice setting. Am J Hosp Palliat Care 22:134-138

80. Davis BA, Porter JB (2000) Long-term outcome of continuous 24-hour deferoxamine infusion via indwelling intravenous catheters in high-risk beta-thalassemia. Blood 95:1229-1236

81. Rossi I, Conan D (1995) Die intramuskuläre Injektion - eine überholte Applikationsart? Schweiz Med Wochenschr 125:1477-1482

82. Domingo P, Mancebo J, Blanch L, Coll P, Martinez E (1994) latrogenic streptococcal meningitis. Clin Infect Dis 19:356-357
83. Griewing B, Machetanz J (2001) Neurologie und Psychiatrie. In: Kramer A, Heeg P, Botzenhart K. Urban \& Fischer Stuttgart: 586

84. Pandian JD, Sarada C, Radhakrishnan VV, Kishore A (2004) latrogenic meningitis after lumbar puncture-a preventable health hazard. J Hosp Infect 56:119-124

85. Torres E, Alba D, Frank A, Diez-Tejedor E (1993) latrogenic meningitis due to Streptococcus salivarius following a spinal tap. Clin Infect Dis 17:525-526

86. Bernau A (1987) Feldstudie intraartikuläre Injektionen. Orthopäd Prax 23:364-385

87. Pal B, Morris J (1999) Perceived risks of joint infection following intra-articular corticosteroid injections: a survey of rheumatologists. Clin Rheumatol 18:264-265

88. Stechow von D, Rittmeister M (2003) Die intraartikuläre Injektion. Orthopädie 32:1127-1135

89. Artunay O, Yuzbasioglu E, Rasier R, Sengul A, Bahcecioglu $H$ (2009) Incidence and management of acute endophthalmitis after intravitreal bevacizumab (Avastin) injection. Eye (Lond) 23:2187-2193

90. Meyer C, Mennel S, Eter N (2007) Endophthalmitisrate mit und ohne topische postoperative Antibiotigagabe nach intraviteraler Avastin-Injektion. Der Ophthalmologe 11:952-957

91. Gonzalez-Michaca L, Chew-Wong A, Soltero L, Gamba G, Correa-Rotter R (2000) Percutaneous kidney biopsy, analysis of 26 years: complication rate and risk factors; comment. Rev Invest Clin 52:125-131

92. Eddleman KA, Malone FD, Sullivan L, Dukes K, Berkowitz RL, Kharbutli Y, Porter TF, Luthy DA, Comstock $\mathrm{CH}$, Saade GR, Klugman S, Dugoff L, Craigo SD, Timor-Tritsch IE, Carr SR, Wolfe HM, D'Alton ME (2006) Pregnancy loss rates after midtrimester amniocentesis. Obstet Gynecol 108:1067-1072

93. Towner D, Currier RJ, Lorey FW, Cunningham GC, Greve LC (2007) Miscarriage risk from amniocentesis performed for abnormal maternal serum screening. Am J Obstet Gynecol 196:608 e601-605; discussion 608 e605

94. Sharma VK, Howden CW (2000) Meta-analysis of randomized, controlled trials of antibiotic prophylaxis before percutaneous endoscopic gastrostomy. Am J Gastroenterol 95:3133-3136 